\title{
Individual susceptibility to neisserial infection?
}

\author{
H. YOUNG, A. B. HARRIS, AND D. H. H. ROBERTSON \\ From the Department of Bacteriology, Edinburgh University Medical School, and the University \\ Department of Venereology, Royal Infirmary, Edinburgh
}

SUMMARY Specimens from genital, anorectal, and pharyngeal sites from 1671 men and 1419 women were cultured for Neisseria gonorrhoeae. Pharyngeal specimens were also cultured for Neisseria meningitidis. $N$. gonorrhoeae was isolated from a genital site $2 \cdot 7$ times more often in men and 1.8 times more often in women who also carried meningococci in their pharynx than from those who did not; the meningococcus was isolated $3 \cdot 4$ times more often from men and $2 \cdot 0$ times more often from women with genital gonorrhoea than from those without. In both men and women the association of each organism with the other was statistically significant $(\mathrm{P}<0.001)$ and may be related to sexual behaviour rather than to individual susceptibility to neisserial infection.

\section{Introduction}

The gonococcus and the meningococcus, the two most important human pathogens in the genus Neisseria, are closely related genetically (Kingsbury, 1967; Kingsbury et al., 1969) and antigenically (Tramont et al., 1974). If there are specific host factors which increase the susceptibility of certain individuals to these organisms, their study could increase our knowledge of the pathogenicity of gonococcal and meningococcal infections.

In an investigation by Willcox et al., (1977) of 150 cultures of throat exudates from patients (predominantly male) who were examined at a clinic for sexually transmitted diseases because they had a history of orogenital intercourse, the meningococcus was isolated nearly six times more often from those with genital gonorrhoea than from those without. In the same study the gonococcus was found 2.5 times more often in those who carried the meningococcus in the pharynx than in those who did not. These workers suggested (Willcox et al., 1977) that confirmation of these findings would support the theory of individual susceptibility to the acquisition of neisseriae.

This paper reports our findings on all patients, male and female, from whom genital (and, in some cases, anorectal) and pharyngeal specimens were cultured during 1977.

Address for reprints: Dr H. Young, Department of Bacteriology, University Medical School, Teviot Place, Edinburgh EH8 9AC; Scotland

Received for publication 23 August 1978

\section{Materials and methods}

Specimens of genital and pharyngeal exudates obtained from 1671 men and 1419 women attending the Department of Venereology at the Royal Infirmary, Edinburgh, during 1977 were cultured in parallel for Neisseria gonorrhoeae. The pharyngeal specimens were also cultured for Neisseria meningitidis.

Urethral and pharyngeal specimens were taken from all male patients in the study while anorectal specimens were also taken for culture from homosexual men. Urethral, cervical, anorectal, and pharyngeal specimens were taken for culture, from all female patients in the study. These patients comprised all known contacts of gonorrhoea. Patients were not routinely asked about recent oral contact, but if such a history was obtained they were included in the study.

Material was inoculated directly on to modified New York City medium (Young, 1978a) at the time of the patient's initial examination. After inoculation, plates were held at $36^{\circ} \mathrm{C}$ in a carbon dioxide-enriched $(10 \%)$ atmosphere and transferred to the laboratory within four hours. Specimens were processed, and $N$. gonorrhoeae and $N$. meningitidis were identified by fluorescent antibody and rapid carbohydrate utilisation tests (Young, 1978b).

Statistical analysis was made by the $\chi^{2}$ method with Yates's correction.

\section{Results}

The results of cultures from the 1671 male and 1419 
female patients are shown in Tables 1 and 2 respectively.

The isolation of gonococi from genital sites is compared with that of meningococei in the pharynx of men and women in Tables 3 and 4 respectively; the isolation of meningococi from the pharynx compared with that of gonococi from genital sites is shown in Tables 5 and 6.

Table 1 Isolation of Neisseria gonorrhoeae from genital and anorectal sites and of Neisseria meningitidis from the pharvnx of 1671 men

\begin{tabular}{|c|c|c|c|}
\hline \multirow[b]{2}{*}{ Genital/anorectal sites } & \multicolumn{3}{|c|}{$\begin{array}{l}\text { Pharyngeal culture results } \\
\text { for N. meningitidis* }\end{array}$} \\
\hline & Positive & Negative & Total \\
\hline \multicolumn{4}{|c|}{ Culture results for $N$. gonorrhoeae } \\
\hline Positive & 89 & 262 & 351 \\
\hline Negative & 97 & 1223 & 1320 \\
\hline Total & 186 & 1485 & 1671 \\
\hline
\end{tabular}

${ }^{*} \chi_{i} ; 89 \cdot 8 ; P<0 \cdot 001$

Table 2 Isolation of Neisseria gonorrhoeae from genital and anorectal sites and of Neisseria meningitidis from the pharynx of 1419 women

\begin{tabular}{|c|c|c|c|}
\hline \multirow[b]{2}{*}{ Genital/anorectal sites } & \multicolumn{3}{|c|}{$\begin{array}{l}\text { Pharyngeal culture results } \\
\text { for N. meningitidis* }\end{array}$} \\
\hline & Positive & Negative & Total \\
\hline \multicolumn{4}{|c|}{ Culture results for 1 . gonorrhoeae } \\
\hline Positive & 96 & 256 & 352 \\
\hline Negative & 148 & 919 & 1067 \\
\hline Total & 244 & 1175 & 1419 \\
\hline
\end{tabular}

${ }^{*} \chi ; 32 \cdot 4 ; \mathrm{P}<0 \cdot 001$

Table 3 Isolation of gonococci from genital and anorectal sites in relation to that of meningococci in the pharynx of 1671 men

\begin{tabular}{lrrr}
\hline & & \multicolumn{2}{l}{$\begin{array}{l}\text { Genital and anorectal } \\
\text { gonorrhoed }\end{array}$} \\
\cline { 3 - 4 } Pharyngeal cultures & No. & No. & $\%$ \\
\hline Meningococci-positive & 186 & 89 & 47.9 \\
Meningococi-negative & 1485 & 262 & 17.6 \\
Total & 1671 & 351 & 21.0 \\
\hline
\end{tabular}

Table 4 Isolation of gonococci from genital and anorectal sites in relation to that of meningococci in the pharynx of 1419 women

\begin{tabular}{lrrr}
\hline & & \multicolumn{2}{l}{$\begin{array}{l}\text { Genital and anorectal } \\
\text { gonorrhoea }\end{array}$} \\
\cline { 2 - 4 } Pharyngeal cultures & No. & No. & $\%$ \\
\hline Meningococci-positive & 244 & 96 & $39 \cdot 3$ \\
Meningococci-negative & 1175 & 256 & $21 \cdot 8$ \\
Total & 1419 & 352 & $24 \cdot 8$ \\
\hline
\end{tabular}

Table 5 Isolation of meningococci from the pharynx in relation to that of gonococci from genital and anorectal sites in 1671 men

\begin{tabular}{lccc}
\hline & & \multicolumn{2}{l}{$\begin{array}{l}\text { Pharyngeal cultures } \\
\text { showing meningococci }\end{array}$} \\
\cline { 3 - 4 } Genital/anorectal cultures & No. & No. & $\%$ \\
\hline Gonococci-positive & 351 & 89 & 25.4 \\
Cionococci-negative & 1320 & 97 & 7.4 \\
Total & 1671 & 186 & $11 \cdot 1$ \\
\hline
\end{tabular}

Table 6 Isolation of meningococci from the pharynx in relation to that of gonococci from genital and anorectal sites in 1419 women

\begin{tabular}{lrrr}
\hline & & \multicolumn{2}{l}{$\begin{array}{l}\text { Pharvngeal cultures } \\
\text { showing meningococci }\end{array}$} \\
\cline { 3 - 4 } Genital/anorectal cultures & No. & No. & $\%$ \\
\hline Gonococi-positive & 352 & 96 & $27 \cdot 3$ \\
Gonococi-negative & 1067 & 148 & $13 \cdot 9$ \\
Total & 1419 & 244 & $17 \cdot 2$ \\
\hline
\end{tabular}

The gonococcus was isolated $2 \cdot 7$ times more often from men and 1.8 times more often from women who were pharyngeal carriers of meningococci than from those who were not (Tables 3 and 4). Conversely, meningococci were' isolated from the pharynx $3 \cdot 4$ times more often from men and $2 \cdot 0$ times more often from women with gonorrhoea than from those without (Tables 5 and 6). The association of each organism with the other is highly significant, in both men $\left(\chi_{1}^{2} 89.08 ; \mathrm{P}<0.001\right)$ and women $\left(\chi_{1}^{2} 32 \cdot 4 ; \mathrm{P}<0 \cdot 001\right)$.

The incidence of pharyngeal gonorrhoea in our study was $5 \cdot 4 \%(19 / 352)$ in women and $3 \cdot 1 \%$ $(11 / 351)$ in men with genital or anorectal gonorrhoea or both; this represents an incidence of $1 \cdot 3 \%$ $(19 / 1419)$ and $0 \cdot 7 \%(11 / 1671)$ of all female and male patients respectively.

\section{Discussion}

Our results confirm the earlier finding (Willcox et al., 1977) that individuals who carry the meningococcus in the pharynx and who have been exposed to the risk of gonorrhoea are more likely to yield positive results to genital culture for $N$. gonorrhoeae and vice versa. Although in the earlier study (Willcox et al., 1977) the meningococcus was isolated approximately six times more often from patients with genital gonorrhoea than from those without, the corresponding factors in our study are lower, $3 \cdot 4$ for men and 2.0 for women. The former six-fold difference (Willcox et al., 1977) reflects a lower meningococcal carriage rate in the group without gonorrhoea $(5 \cdot 3 \%)$ and a higher rate in the group 
with genital gonorrhoea $(31 \cdot 3 \%)$ than the corresponding carriage rates in our study $-7 \cdot 4 \%$ and $13.9 \%$ in men and women without gonorrhoea and $25.4 \%$ and $27 \cdot 3 \%$ in those with gonorrhoea respectively. Our finding that the gonococcus was isolated 2.7 times more often from men and 1.8 times more often from women who were pharyngeal carriers of the meningococcus than from those who were not, is in good agreement with the $2 \cdot 5$-fold difference quoted previously (Willcox et al., 1977).

Thus, in our study each organism was isolated two to three times more frequently in the presence of the other. The factors responsible for this association are unknown. It has been reported (Foster and Labrum, 1976) that there is an increased risk of gonococcal infection in blood group B subjects, and other workers (Miler et al., 1977) confirmed this for white patients but not for West Indians. It was concluded (Miler et al., 1977) that such data must be carefully collected on well-defined ethnic groups over a range of samples before a definite conclusion on intrinsic suseptibility to gonococcal infection based on blood group differences can be made. Analysis of the results by blood group or ethnic group was not attempted in the present study.

Another possibility is that our results, and those of Willcox et al. (1977), do not indicate that specific host factors are involved in individual susceptibility but rather reflect that the more frequent acquisition of both organisms is related to sexual behaviour. It has been suggested (World Health Organisation, 1978) that more frequent changes of sexual partner may have contributed to the increase in gonorrhoea found in many countries in recent years. Assuming that an intimate activity, such as kissing, is a common prelude to sexual intercourse, more frequent changes of partner might increase the chance of acquiring meningococci in the pharynx.
If the population examined in this study includes a group whose carriage of neisseriae merely reflects the association of intimate behaviour with the exchange of flora, then it might be worthwhile to investigate the carriage of other 'marker' organisms, for example, Streptococcus pyogenes and Haemophilus influenzae. If oropharyngeal carriage of these organisms indicates a similar correlation with genital gonorrhoea then the postulated host susceptibility factor for neisseriae is unlikely.

We thank Professor J. G. Collee for constructive criticism and helpful advice in the preparation of this paper.

\section{References}

Foster, M. T. and Labrum, A. H. (1976). Relation of infection with Neisseria gonorrhoeae to ABO blood groups. Journal of Infectious Diseases, 133, 329-330.

Kingsbury, D. T. (1967). Deoxyribonucleic acid homologies among species of the genus Neisseria. Journal of Bacteriology, 94, 870-874.

Kingsbury, D. T., Fanning, G. R., Johnson, K. E., and Brenner, D. J. (1969). Thermal stability of interspecies Neisseria DNA duplexes. Journal of General Microbiology, 55, 201-208.

Miler, J. J., Novotny, P., Walker, P. D., Harris, J. R. W., and Maclennan, I. P. B. (1977). Neisseria gonorrhoeae and ABO isohaemagglutinins. Infection and Immunity, 15, 713-719.

Tramont, E. S., Sadoff, J. C., and Artenstein, M. S. (1974). Crossreactivity of Neisseria gonorrhoeae and Neisseria meningitidis and the nature of antigens involved in the bactericidal reaction. Journal of Infectious Diseases, 130, 240-247.

Willcox, R. R., Spencer, R. S., and Ison, C. (1977). Which Neisseria? British Journal of Venereal Diseases, 53, 394-397.

World Health Organisation (1978). Neisseria gonorrhoeae and gonococcal infections. Technical Report Series 616. WHO: Geneva.

Young, H. (1978a). Cultural diagnosis of gonorrhoea with modified New York City (MNYC) medium. British Journal of Venereal Diseases, 54, 36-40.

Young, H. (1978b). Identification and penicillinase testing of Neisseria gonorrhoeae from primary isolation cultures on modified New York City medium. Journal of Clinical Microbiology, 7, 247-250. 